\title{
Influenza from scratch
}

Influenza A virus has been reconstructed entirely from cloned cDNA segments. Until now, the virus had been notoriously difficult to alter in vitro because the genome is distributed among eight subgenomic segments of negative-stranded RNA. Researchers have relied on the use of helper viruses to study the pathogen, but such approaches allow alteration of only a small subset of influenza virus genes. In the new work, published in Proc. Natl. Acad. Sci. USA (96, 9345-9350, 1999), scientists have transfected human cell lines with eight separate plasmids, each carrying the cDNA of a subgenomic segment in front of an RNA polymerase I promoter. The approach yielded highly concentrated virus stocks, and the CDNA plasmids were amenable to alteration by standard recombinant DNA techniques. Yoshihiro Kawaoka, a senior author on the paper, cautions that genetic engineering of influenza should be approached carefully: "Before initiating such experiments, scientists should weigh the risks and benefits of the studies and the containment levels at which the work should be performed." Historically, new strains of influenza have periodically caused deadly pandemics. Similar work by the teams of George Brownlee and Peter Palese will soon be published in the J. Virology (73, in press, 1999). Influenza induces strong mucosal immunity and does not integrate into the host genome, making it a potentially useful vector for both vaccines and gene therapy.

\section{AAV muscles ahead}

Scientists from ARIAD Pharmaceuticals (Cambridge, MA) and Stanford University (Stanford, CA) have shown that adeno-associated viral (AAV) vectors significantly outperform adenoviral vectors when used to transfect muscle cells with an inducible gene expression system. In a head-to-head comparison, the scientists used the two vectors to transfect mice muscle cells with a construct containing the gene for human growth hormone (hGH) under the control of a rapamycin-inducible expression system (Proc. Natl. Acad. Sci. USA 96, 8657-8662, 1999). Injection of rapamycin into mouse muscle was shown to rapidly induce hGH synthesis, which gradually declined as rapamycin levels diminished. Importantly, expression of hGH remained stable when AAV was used as vector, but progressively declined when genes were transferred using the adenoviral vector. According to Victor Rivera, corresponding author on the study, AAV integrates its genes into infected host cells and thus avoids immune responses induced by adenovirus that eventually kill cells carrying the foreign gene. "We tried both systems," says Rivera, "and our results demonstrated we should fully explore AAV." He contends that the ease and efficiency of the rapamycin system should make it broadly applicable.

Research News Briefs written by Alan Dove and Holger Breithaupt

\section{Aptamers on the scaffold}

In independent reports published in Proc. Natl. Acad. Sci. USA 6, 8567-8572, 1999) and Science $(285,591-595,1999)$, two teams of researchers have recently described nearly identical approaches for identifying novel components of cellular signaling pathways, technology that should prove useful both in basic research and the identification of new drug targets. Each group screened yeast cells expressing a library of aptamers - short random peptide sequences expressed on a larger protein scaffold-to identify peptides that inhibit specific intracellular signals in yeast. Once inhibitory peptides had been identified, they were used as "bait" in two-hybrid screens to determine which yeast gene products they were binding. The approach identified new components of the yeast mating pheromone signaling pathway and the mitotic spindle checkpoint. Having proven the concept, the approach could conceivably be employed for signaling studies and drug target identification in mammalian cells, which lack the power of yeast genetics. While emphasizing that several hurdles remain before the technique becomes standard practice, Roger Brent, senior author on the PNAS paper, says that "if the two-hybrid experience is a guide, I would guess this is about a year away from widespread use by relatively unsophisticated laboratories."

\section{In silico matchmaking}

A computer algorithm has been described that may serve as a prototype for in silico approaches that assign function to hitherto unknown proteins. The method, described in Science $(285,751-753,1999)$, is based on the observation that pairs of interacting proteins often have orthologs in other organisms in which the two proteins are fused into a single chain. Sequence homologies with different segments of a protein from another organism can therefore be used to predict an interaction between two different proteins. Applying the algorithm to the 4290 known protein from Escherichia coli, David Eisenberg and his colleagues came up with 6809 putative interacting or functional-related protein pairs in the bacteria. A similar search resulted in 45,502 similar patterns for the 5,800 yeast proteins. Although the approach yielded a high proportion $(82 \%)$ of false positives, Eisenberg expects that refinements can improve the reliability of the method, particularly by eliminating homologies like nucleotide-binding cassettes. "Learning how to filter these domains out is very important," he says. Together with his colleague Todd Yeates, Eisenberg has founded Protein Pathways (Los Angeles, CA) to commercialize the method.

\section{Retargeting tropism}

German and French scientists have successfully redirected the cellular tropism of adeno-associated virus type 2 (AAV2) by displaying short peptides on the viral capsid that are recognized by specific receptors on target cells. By studying the uptake of AAV2 strains in which a 14-amino-acid ligand, L14, had been inserted into six different domains of the capsid, they showed that one strain successfully infects AAV2-resistant cells displaying L14-specific integrin on its surface. Further experiments demonstrated that other ligands up to 34 amino acids in length can also be inserted into the AAV2 capsid. Though the specificity of the AAV2-integrin receptor interaction in this study was not fully characterized, the researchers think that their approach could be widely applicable in the design of vectors specific for other cell types. "These studies demonstrate that, in principle, it is possible to redirect AAV2," says Anne Girod, the lead author on the article (Nat. Med. 9, 1052-1056, 1999). The team's next aim is to find peptides that allow vectors to be targeted to specific cells types in vivo. According to Girod, modified AAV2 vectors that specifically infect T-lymphocytes could be an early application. 